\title{
Selection of antibiotics suppressing bacterial and mycoplasma contamination of the original explants during in vitro microclonal propagation of grape \\ Sadigova E.E., Garagozov T.H.
}

Institute of Molecular Biology and Biotechnology, Azerbaijan National Academy of Sciences, Baku, Azerbaijan E-mail:bioloq.2015@mail.ru

Key message. The research was conducted for the selection of antibiotics and determination of their effects on obtaining donor plants in vivo, improvement of morphogenesis and creation of plant collections with qualitative characteristics for in vitro microclonal propagation.

Keywords: antibiotics, in vitro, microclonal propagation

The object of the study was an endangered, ancient, aboriginal grape variety Shirvan-Shahi. The research aimed to select antibiotics and determine their effects on obtaining donor plants in vivo, improvement of morphogenesis and creation of plant collections with qualitative characteristics for in vitro microclonal propagation. For this purpose, annual seedlings were treated with IMA (indolylbutyric acid) $(50 \mathrm{mg} / \mathrm{l})$ along with antibiotics and then cultivated in a liquid medium, as well as in various substrates for rooting. In all stages of obtaining root plants from one-eyed cuttings, antibiotics were used both separately and in various proportions. The plant was transferred to a sterile soil mixture and cultivated under artificial climate conditions. The plant treated with antibiotics became the source of primary explants in the first stages of in vitro microclonal propagation.

Among the studied antibiotics, ceftriaxone appeared to be effective in all stages of the study in the survival of cuttings in vivo and plant regeneration in vitro. The obtained results convincingly showed the effectiveness of using antibiotics at a concentration of $10 \mathrm{mg} / \mathrm{l}$ to obtain the initial material for in vitro microclonal propagation. Ceftriaxone at concentrations of 10 $\mathrm{mg} / \mathrm{l}$ did not cause inhibition of growth processes in the initial plants. The antibacterial therapy of the initial material significantly increased morphogenesis in the first stages of in vitro microclonal propagation. 